\title{
Berechnung kinetischer Isotopeneffekte für die Reaktion von Methyljodid mit Hydroxid-Ion*
}

\author{
A. V. Willi \\ Chemistry Department, Brookhaven National Laboratory, Upton, N.Y., USA ** \\ und Columbia University, College of Pharmaceutical Sciences, \\ 115 W 68th Street, New York, N.Y. 10023, USA *** \\ (Z. Naturforschg. 21 a, 1385-1390 [1966] ; eingegangen am 20. Mai 1966)
}

\begin{abstract}
Kinetic carbon-13, deuterium, and oxygen-18 isotope effects are calculated from force constants for the $\mathrm{S}_{\mathrm{N}} 2$ reaction of $\mathrm{CH}_{3} \mathrm{I}$ with $\mathrm{OH}^{-}$. The secular equations for the vibrational frequencies of reactants and transition states are solved on an IBM 7094 computer. Values for 7 force constants of the planar $\mathrm{CH}_{3}$ moiety in the transition state (with an $\mathrm{sp}^{2}$ atom) are obtained by comparison with suitable stable molecules. The stretching force constants $f_{\mathrm{CO}}=f_{11}, f_{\mathrm{CI}}=f_{22}$, and $f_{12}$ in the transition state are chosen as linear functions of $x$, the degree of bond making and bond breaking, with the aid of eqs. (1) and (2).

The carbon isotope effect as a function of $x$ exhibits a flat maximum near $x=0.6$. If the bending force constants $f_{\mathrm{HCO}}$ and $f_{\mathrm{HCI}}$ are kept constant, $k_{12} / k_{13}$ is significantly lower than its maximum value only for values of $x \leqq 0.3$ and $x \geqq 0.8$. There is only a small influence of $x$ on the deuterium isotope effect, $k_{\mathrm{H}} / k_{\mathrm{D}}$ (compare rates of $\mathrm{CH}_{3} \mathrm{I}$ and $\mathrm{CD}_{3} \mathrm{I}$ ). However, $k_{\mathrm{H}} / k_{\mathrm{D}}$ is strongly dependent on $f_{\mathrm{HCO}}$ and $f_{\mathrm{HCI}}$. The oxygen-18 isotope effect decreases with increasing $x$. Experimental data of the D isotope effect - if they were available - could be used to adjust the bending force constants $f_{\mathrm{HCO}}$ and $f_{\mathrm{HCI}}$ ( - or to adjust $f_{\mathrm{HCO}}$ if $f_{\mathrm{HCI}}$ is held constant). The degree of unsymmetry of the transition state could then be estimated with the aid of the $\mathrm{C}$ isotope effect. Experimental data of the $O$ isotope effect would supply information on the direction of the unsymmetry - which is needed for a distinction between 2 possible values of $x-$. There is approximate agreement between the calculated and the experimental temperature dependence of the carbon-13 isotope effect.
\end{abstract}

Berechnungen kinetischer Isotopeneffekte im Vergleich zu experimentellen Daten sind bisher für die nucleophilen Substitutionsreaktionen von Methyljodid mit Jodid-Ion ${ }^{1}$ und mit Cyanid-Ion ${ }^{2}$ sowie für die Solvolyse von Methyljodid in Wasser ${ }^{1}$ durchgeführt worden. Im Anschluß an jene Arbeiten wird hier nun zur Reaktion von Methyljodid mit Hydroxid-Ion übergegangen. Es handelt sich um vollständige Berechnungen aller Schwingungsfrequenzen von Ausgangsverbindungen und Übergangszuständen sowie von Isotopen-substituierten Ausgangsverbindungen und Übergangszuständen durch Lösung der Säkulargleichung in einer elektronischen Rechenanlage IBM 7094. Die Frequenzen - sowie die Partikel-Massen und Trägheitsmomente - werden dann in die allgemeine Gleichung für den Isotopen-

* Im November 1964 auszugsweise vorgetragen im OrganischChemischen Kolloquium der Universität München sowie in den Kolloquien des Institutes für Kernchemie der Technischen Hochschule Darmstadt und des Institutes für Strahlenchemie des Kernforschungszentrums Karlsruhe.

** Diese Arbeit wurde unter den Auspizien der U.S. Atomenergie-Kommission durchgeführt.

** Gegenwärtige Adresse. (Für einen Auslandsaufenthalt beurlaubt von der Philosophisch-Naturwissenschaftlichen Fakultät der Universität Bern, Schweiz.)

1 A. V. Willi, Can. J. Chem. 44 [1966], im Druck.

2 A. V. Willi, Z. Naturforschg. 21 a, 1377 [1966]. effekt eingesetzt, wobei entweder die primäre Form oder die durch Anwendung der Produktregel umgewandelte Form benutzt wird $^{3}$. Die Grundlage bildet somit die Theorie des Übergangszustandes. Wechselwirkungen mit dem Lösungsmittel, Anharmonizitätseinflüsse auf die Schwingungen und der wellenmechanische Tunneleffekt werden nicht berücksichtigt. Derartige Berechnungen mit Kraftkonstanten, Atommassen und geometrischen Angaben als Ausgangsdaten sind bereits von verschiedenen Autoren ausgeführt und auf allgemeine und spezielle Probleme angewandt worden ${ }^{4-7}$.

\section{Grundlagen der Berechnung}

Aus dem Vergleich berechneter und experimenteller Isotopeneffekte ergeben sich Zahlenwerte für

3 L. Melander, Isotope Effects on Reaction Rates, Ronald Press, New York 1960.

4 H. S. Johnston, Science 128, 1145 [1958]. - T. E. Sharp u. H. S. Johnston, J. Chem. Phys. 37, 1541 [1962].

5 M. Wolfsberg u. M. J.Stern, Pure Appl. Chem. 8, 225, 325 [1964]. - M. J. Stern u. M. Wolfsberg, J. Chem Phys. 39, 2776 [1963].

6 M. J. Goldstein u. G. L. Thayer, J. Am. Chem. Soc. 87, 1933 [1965].

7 A. V. Willi, Z. Naturforschg. 16 a, 838 [1961]; Helv. Chim. Acta 47, 647, 655, 837 [1964]. 
Kraftkonstanten im Übergangszustand, die als (relative und qualitative) Aussagen über die Bindungsverhältnisse im betreffenden System betrachtet werden dürfen. In dieser Arbeit werden die von Lynv und YANkwich $^{8}$ im Temperaturbereich von 284,6 bis $331,2^{\circ} \mathrm{K}$ gemessenen Kohlenstoff-13-Isotopeneffekte auf die Reaktion von $\mathrm{CH}_{3} \mathrm{~J}$ mit $\mathrm{OH}^{-}$verwendet $\left(k_{12} / k_{13}=1,0355\right.$ bei $\left.284,6^{\circ}\right)$. Bei Umrechnung des von Bender und Hoeg ${ }^{9}$ bei $298,2{ }^{\circ} \mathrm{K}$ gemessenen Kohlenstoff-14-Isotopeneffektes erhält man einen etwas höheren Wert für $k_{12} / k_{13}$.

Bei Durchführung der Berechnungen wird für Methyljodid wiederum der gleiche Satz von 13 Kraftkonstanten benutzt wie in der vorhergehenden Arbeit, - ebenso sind die geometrischen Daten die gleichen ${ }^{2}$. Die Ausgangsdaten für Hydroxid-Ion und für den Übergangszustand $\mathrm{J}_{\ldots} \mathrm{CH}_{3} \ldots \mathrm{OH}$ sind in Tab. 1 zusammengefaßt. Die ebene $\mathrm{CH}_{3}$ -

\begin{tabular}{|c|c|c|}
\hline & $\mathrm{OH}^{-}$ & {$\left[\mathrm{J} \ldots \mathrm{CH}_{3} \ldots \mathrm{OH}\right] \neq$} \\
\hline$r_{\mathrm{CH}}$ & & $1,08 \AA$ \\
\hline$r_{\mathrm{CJ}}$ & & $2,35 \AA$ \\
\hline$r_{\mathrm{CO}}$ & & $1,57 \AA$ \\
\hline$r_{\mathrm{OH}}$ & $0,96 \AA$ & $0,96 \AA$ \\
\hline$\Varangle \mathrm{HCH}$ & & $120^{\circ}$ \\
\hline$\Varangle \mathrm{HCJ}$ & & $90^{\circ}$ \\
\hline$\Varangle \mathrm{HCO}$ & & $90^{\circ}$ \\
\hline$\Varangle \mathrm{COH}$ & & $\begin{array}{l}108,9^{\circ} \\
5,3 \text { oder } 5,05 \mathrm{mdvn} / \AA \AA\end{array}$ \\
\hline $\begin{array}{l}f_{\mathrm{CH}} \\
f_{\mathrm{CJ}}\left(=F_{22}\right)\end{array}$ & & $\underset{*}{5,3} \underset{\text { oder }}{5,05} \mathrm{mdyn} / \AA$ \\
\hline $\begin{array}{l}f_{\mathrm{CO}}\left(=F_{11}\right) \\
f_{12}\left(=F_{12}\right)\end{array}$ & & $\begin{array}{l}* \\
*\end{array}$ \\
\hline$f_{\mathrm{OH}}$ & $\stackrel{7,3}{7}$ & 7,45 mdyn/ $\AA$ \\
\hline$f_{\mathrm{HCH}}$ & & $\begin{array}{c}0,36 \text { oder } 0,525 \mathrm{mdyn} / \AA \\
* \\
*\end{array}$ \\
\hline$f_{\mathrm{HCO}}$ & & $0,144 \mathrm{mdyn} \AA$ \\
\hline$f_{\mathrm{COH}}$ & & $\begin{array}{l}0,02 \text { oder } 0,40 \mathrm{mdyn} \AA \\
0,30 \text { mdyn } \AA\end{array}$ \\
\hline
\end{tabular}

* Die Werte der mit einem Stern versehenen Kraftkonstanten sind hier noch offen.

Tab. 1. Geometrische Daten und Kraftkonstanten.

Gruppierung im Übergangszustand soll wiederum auf der JCO-Achse senkrecht stehen, wobei die CJund CO-Abstände $10 \%$ länger sein sollen als in entsprechenden stabilen Bindungen. Die Begründung

8 K. R. Lynn u. P. E. Yankwich, J. Am. Chem. Soc. 83, 53, 790 [1961].

9 M. L. Bender u. D. F. Hoeg, J. Am. Chem. Soc. 79, 5649 [1957].

10 L. H. Jones, J. Chem. Phys. 22, 217 [1954].

11 W. R. Busing u. D. F. Hornig, J. Phys. Chem. 65, 284 [1961]. der für die $\mathrm{CH}_{3}$-Gruppierung eingesetzten $7 \mathrm{Kraft}$ konstanten ist aus der vorhergehenden Arbeit zu ersehen ${ }^{2}$. Wie beim Methanol beträgt der $\mathrm{COH}$ Winkel des Übergangszustand-Modells $108,9^{\circ}$, wobei die OH-Bindung in der Symmetrieebene zwischen zwei CH-Bindungen der $\mathrm{CH}_{3}$-Gruppierung liegt. Als Grundlage zur Wahl der Kraftkonstante $f_{\mathrm{OH}}$ im Hydroxid-Ion dienen Literaturangaben über an LiOH-Kristallen ${ }^{10}$ und an wäßrigen KOH-Lösungen ${ }^{11}$ gemessenen Frequenzen. Der für den Übergangszustand eingesetzte Wert von $f_{\mathrm{OH}}$ ist das arithmetische Mittel der Werte für Hydroxid-Ion und Wasser bzw. Methanol $\left(f_{\mathrm{OH}}=7,6 \mathrm{mdyn} / \AA\right)^{12}$. Die $\mathrm{COH}$-Deformationskraftkonstante wird in der Mehrzahl der Proberechnungen als klein angenommen $\left(f_{\mathrm{COH}}=0,02 \mathrm{mdyn} \AA\right)$, so $\mathrm{da} \beta$ sich die zugehörige Eigenschwingung annähernd wie ein klassischer Oszillator verhält. Der Wirklichkeit näher sollte jedoch der in einigen Fällen benutzte Wert von $f_{\mathrm{COH}}=$ $0,40 \mathrm{mdyn} \AA$ sein, der etwa halb so groß ist wie die betreffende Deformationskraftkonstante in Methanol. (Aus $1340 \mathrm{~cm}^{-1}$ als COH-Deformationsfrequenz für $\mathrm{CH}_{3} \mathrm{OH}^{12}$ ergibt sich überschlagsmäßig $f_{\mathrm{COH}}=$ 0,81 mdynÅ.) Die hier berechneten Isotopeneffekte sind allerdings nur wenig abhängig von der speziellen Wahl von $f_{\mathrm{COH}}$. Der für die Torsionskraftkonstante $f_{\mathrm{HCOH}}$ des Übergangszustandes eingesetzte Wert (Verdrehung der OH-Bindung um die COAchse relativ zu den CH-Bindungen) ist aus der an Methanol gemessenen Rotationshemmung abgeleitet ${ }^{13}$. Die potentielle Energie wird hier als Funktion einer Torsionskoordinate formuliert (mit den vier Atomen $\mathrm{HCOH}$ des Dieder-Winkels in der Symmetrieebene).

Wie aus Tab. 1 zu ersehen, sind nun noch 5 Kraftkonstanten frei wählbar, nämlich $f_{\mathrm{CO}}=F_{11}, f_{\mathrm{CJ}}=F_{22}$, $f_{12}=F_{12}, f_{\mathrm{HCO}}$ und $f_{\mathrm{HCJ}}$. Es besteht die einschränkende Bedingung:

$$
f_{\mathrm{CO}} f_{\mathrm{CJ}}-f_{12}^{2}=d \leqq 0 .
$$

Ein positives $d$ würde keinem Übergangszustand, sondern einem in bezug auf alle Koordinaten stabilen System entsprechen.

\footnotetext{
12 G. Herzberg, Infrared and Raman Sectra of Polyatomic Molecules, Van Nostrand, New York 1945.

13 W. G. F f ately u. F. A. Miller, Spectrochim. Acta 19, 614 [1963]. - D. G. Burkhard u. D. M. Dennison, J. Mol. Spectr. 3, 299 [1959].
} 


\section{Ergebnisse und Diskussion}

\section{Die Kohlenstoff-, Deuterium- und Sauerstoff- Isotopeneffekte}

Von den mehr als 200 ausgeführten Proberechnungen wird in den Tabellen 2 und 3 eine kleine Auswahl gegeben. Die Rechnungen Nr. 8, 16 und 100 in Tab. 2 bilden Beispiele für den Grenzfall mit $d=0$ und $v_{\mathrm{L}}^{\neq}=0$. (Das letztere heißt nicht, daß die Frequenz der Übergänge über den Potentialberg gleich Null sei, sondern bedeutet, daß es sich beim Durchschreiten des Übergangszustandes in der Re. aktionskoordinate um eine aperiodische Bewegung handelt.) In Nr. 8 (Tab. 2) ist der berechnete Kohlenstoff-Isotopeneffekt $k_{12} / k_{13}$ zu hoch. Eine Anpassung an den experimentellen Wert ist erreichbar durch Erhöhung der Deformationskraftkonstanten $f_{\mathrm{HCO}}$ und $f_{\mathrm{HCJ}}(\mathrm{Nr} .16)$. Geht man zu negativen $d$ und imaginären $\nu_{\mathrm{L}}^{\neq}$über, indem man $f_{12}$ erhöht, so steigt $k_{12} / k_{13}$ (vgl. Nr. 8 und 4, 16 und 11, 100 und 13). Eine theoretische Diskussion dieser Erscheinung wird in der vorhergehenden Arbeit gegeben ${ }^{2}$. Die Deformationskraftkonstante $f_{\mathrm{HCO}}$ ist jetzt zur Anpassung an den experimentellen ${ }^{13} \mathrm{C}$ Isotopeneffekt noch weiter $\mathrm{zu}$ erhöhen. In allen folgenden Proberechnungen wird nun $f_{\mathrm{HCJ}}$ (willkürlich) auf 0,205 mdyn $\AA$ konstant gehalten, was etwas tiefer ist als der angepaßte Wert im symmetrischen System J ... $\mathrm{CH}_{3} \ldots{ }^{1}$. Die entscheidende Größe ist im wesentlichen die Summe von $f_{\mathrm{HCO}}$ und
$f_{\mathrm{HCJ}}$. Eine andere Möglichkeit zur Wahl der Kraftkonstanten $f_{\mathrm{CO}}, f_{\mathrm{CJ}}$ und $f_{12}$ bietet die Anwendung von Gl. (7) der vorhergehenden Arbeit, wobei ebenfalls negative $d$ erhalten werden. Auf diese Weise ergeben sich die Daten der Rechnungen Nr. 104, 109, 134 und 203 aus $f_{\mathrm{CO}}^{\prime}=2,0, f_{\mathrm{CJ}}^{\prime}=1,0$ und $f_{\neq}=7,0 \mathrm{mdyn} / \AA$. Hierbei ist $f_{\mathrm{HCO}}$ sogar auf 1,2 bzw. 1,3 mdyn $\AA$ anzupassen, jenachdem welcher Wert für $f_{\mathrm{CH}}$ eingesetzt wird (Nr. 109 und 134) ${ }^{13 a}$. Der Vergleich der Rechnungen Nr. 109 und 203 zeigt, daß die Erhöhung von $f_{\mathrm{COH}}$ von 0,02 auf $0,40 \mathrm{mdyn} \AA$ den ${ }^{13} \mathrm{C}$-Isotopeneffekt nur geringfügig und den D-Isotopeneffekt praktisch gar nicht beeinflußt. Der O-Isotopeneffekt sinkt dabei von $k_{016} / k_{018}=0,9855$ in Nr. 109 auf 0,9795 in Nr. 203.

Der Deuterium-Isotopeneffekt $k_{\mathrm{H}} / k_{\mathrm{D}}$ (Vergleich der Reaktionsgeschwindigkeiten von $\mathrm{CH}_{3} \mathrm{~J}$ und $\mathrm{CD}_{3} \mathrm{~J}$ ) wird (abgesehen von der Abhängigkeit von $f_{\mathrm{CH}}$ und $\left.f_{\mathrm{HCH}}\right)$ im wesentlichen durch die Größe der Deformationskraftkonstanten $f_{\mathrm{HCO}}$ und $f_{\mathrm{HCJ}}$ bestimmt und sinkt bei deren Erhöhung. Der Übergang zu negativen $d$ und imaginären $\nu_{\mathrm{L}}^{\neq}$bewirkt ein schwaches Absinken von $k_{\mathrm{H}} / k_{\mathrm{D}}$ (siehe Nr. 16 und 11, sowie 100 und 13) - ebenso wie bei den Reaktionen von $\mathrm{CH}_{3} \mathrm{~J}$ mit $\mathrm{J}^{-1}$ und mit $\mathrm{CN}^{-2}$. Das ist auf die veränderten Kopplungsverhältnisse der Schwingungen zurückzuführen ${ }^{1,5}$. Bei der Bewegung des zentralen C-Atoms vom Energiemaximum herunter bleiben nun die H-Atome hinter dem C-Atom zurück, denn es wird genügend Energie frei zur Überwindung der Rückstellkräfte.

\begin{tabular}{|c|c|c|c|c|c|c|c|c|c|c|c|c|c|}
\hline \multirow{2}{*}{ Nr. } & \multicolumn{7}{|c|}{ Utbergangszustands-Kraftkonstanten } & \multirow[b]{2}{*}{$v_{\mathrm{L}}^{ \pm}, 12$} & \multirow[b]{2}{*}{$v_{\mathrm{L}}^{\mathrm{t}}, 12 / v_{\mathrm{L}}^{\neq}, 13$} & \multirow[b]{2}{*}{$\begin{array}{c}\left(284,6^{\circ}\right) \\
k_{12} / k_{13}\end{array}$} & \multirow[b]{2}{*}{$\begin{array}{c}\left(298,2^{\circ}\right) \\
k_{\mathrm{H}} / k_{\mathrm{D}}\end{array}$} & \multicolumn{2}{|c|}{$\begin{array}{l}\text { ARRHENIUS-Para- } \\
\text { meter von } k_{12} / k_{13}\end{array}$} \\
\hline & $f_{\mathrm{CO}}$ & $f_{\mathrm{CJ}}$ & $f_{12}$ & $f_{\mathrm{CH}}$ & $f_{\mathrm{HCO}}$ & $f_{\mathrm{HCJ}}$ & $f_{\mathrm{COH}}$ & & & & & A & $(A Z P E)$ \\
\hline 8 & 0,2 & 0,2 & 0,2 & 5,3 & 0,010 & 0,010 & 0,020 & 0 & 1,0296 & 1,0577 & & 1,0137 & 1,0434 \\
\hline 16 & 0,2 & 0,2 & 0,2 & 5,3 & 0,800 & 0,205 & 0,020 & 0 & 1,0296 & 1,0349 & 0,603 & 1,0203 & 1,0142 \\
\hline 100 & 0,2 & 0,2 & 0,2 & 5,3 & 1,000 & 0,205 & 0,020 & 0 & 1,0296 & 1,0313 & 0,491 & & \\
\hline 4 & 0,2 & 0,2 & 1,5 & 5,3 & 0,010 & 0,010 & 0,020 & $620 \mathrm{i}$ & 1,0309 & 1,0638 & & 1,0126 & 1,0505 \\
\hline 11 & 0,2 & 0,2 & 1,5 & 5,3 & 0,800 & 0,205 & 0,020 & $608 \mathrm{i}$ & 1,0287 & 1,0385 & 0,568 & & \\
\hline 13 & 0,2 & 0,2 & 1,5 & 5,3 & 1,000 & 0,205 & 0,020 & $608 \mathrm{i}$ & 1,0286 & 1,0346 & 0,463 & 1,0187 & 1,0156 \\
\hline 104 & 0,25 & $-0,75$ & 1,75 & 5,3 & 1,000 & 0,205 & 0,020 & $738 \mathrm{i}$ & 1,0302 & 1,0387 & & & \\
\hline 109 & 0,25 & $-0,75$ & 1,75 & 5,3 & 1,200 & 0,205 & 0,020 & $737 \mathrm{i}$ & 1,0300 & 1,0350 & 0,3766 & 1,0199 & 1,0148 \\
\hline 134 & 0,25 & $-0,75$ & 1,75 & 5,05 & 1,300 & 0,205 & 0,020 & $736 \mathrm{i}$ & 1,0300 & 1,0351 & 0,397 & 1,0200 & 1,0148 \\
\hline 203 & 0,25 & $-0,75$ & 1,75 & 5,3 & 1,200 & 0,205 & 0,400 & $736 \mathrm{i}$ & 1,0301 & 1,0340 & 0,3764 & & \\
\hline
\end{tabular}

Tab. 2. Berechnete Isotopeneffekte. Die Werte der hier nicht erwähnten Kraftkonstanten sind die gleichen wie in Tab. 1. Valenzkraftkonstanten in mdyn/ $\AA$ und Deformationskraftkonstanten in mdyn $\AA . \nu_{\mathrm{L}}^{ \pm}$in $\mathrm{cm}^{-1}$.

13a In allen Beispielen mit imaginären $\nu_{\mathrm{L}}^{\neq}$wird $k_{12} / k_{13}$ vermutlich noch durch den Beitrag eines schwachen Tunneleffektes um einige Promille erhöht. Da sich dieser Beitrag jedoch schlecht genauer berechnen läßt, wird er hier bei den Anpassungen von $k_{12} / k_{13}$ auf den experimentellen Wert vernachlässigt. 


\begin{tabular}{|c|c|c|c|c|c|c|c|c|c|c|}
\hline \multirow{2}{*}{ Nr. } & \multicolumn{5}{|c|}{ Utbergangszustands-Kraftkonstanten } & \multirow{2}{*}{$\begin{array}{c}\left(284,6^{\circ}\right) \\
k_{12} / k_{13}\end{array}$} & \multirow{2}{*}{$A$} & \multirow{2}{*}{$(A Z P E)$} & \multirow{2}{*}{$\begin{array}{c}\left(298,2^{\circ}\right) \\
k_{\mathrm{H}} / k_{\mathrm{D}}\end{array}$} & \multirow{2}{*}{$k_{016} / k_{018}$} \\
\hline & $x$ & $f_{\mathrm{CH}}$ & $f_{\mathrm{HCH}}$ & $f_{\mathrm{HCO}}$ & $f_{\mathrm{COH}}$ & & & & & \\
\hline 137 & 0,05 & 5,3 & 0,36 & 1,000 & 0,02 & 1,0194 & 1,0108 & 1,0085 & 0,452 & 1,0110 \\
\hline 138 & 0,10 & 5,3 & 0,36 & 1,000 & 0,02 & 1,0239 & 1,0126 & 1,0112 & 0,452 & 1,0059 \\
\hline 139 & 0,15 & 5,3 & 0,36 & 1,000 & 0,02 & 1,0269 & & & 0,452 & 1,0020 \\
\hline 140 & 0,20 & 5,3 & 0,36 & 1,000 & 0,02 & 1,0293 & 1,0149 & 1,0141 & 0,452 & 0,9986 \\
\hline 141 & 0,30 & 5,3 & 0,36 & 1,000 & 0,02 & 1,0328 & & & 0,453 & \\
\hline 170 & 0,40 & 5,3 & 0,36 & 1,000 & 0,02 & 1,0354 & & & 0,456 & 0,9862 \\
\hline 101 & 0,50 & 5,3 & 0,36 & 1,000 & 0,02 & 1,0373 & 1,0199 & 1,0171 & 0,460 & 0,9794 \\
\hline 118 & 0,60 & 5,3 & 0,36 & 1,000 & 0,02 & 1,0379 & 1,0206 & 1,0170 & 0,464 & 0,9721 \\
\hline 119 & 0,70 & 5,3 & 0,36 & 1,000 & 0,02 & 1,0363 & & & 0,469 & 0,9650 \\
\hline 107 & 0,80 & 5,3 & 0,36 & 1,000 & 0,02 & 1,0306 & 1,0196 & 1,0108 & & \\
\hline 120 & 0,85 & 5,3 & 0,36 & 1,000 & 0,02 & 1,0257 & & & 0,472 & 0,9592 \\
\hline 102 & 0,90 & 5,3 & 0,36 & 1,000 & 0,02 & 1,0191 & 1,0183 & 1,0008 & 0,471 & 0,9596 \\
\hline 103 & 0,95 & 5,3 & 0,36 & 1,000 & 0,02 & 1,0102 & & & 0,469 & 0,9615 \\
\hline 142 & 0,05 & 5,3 & 0,36 & 0,250 & 0,02 & 1,0355 & 1,0078 & 1,0288 & 1,077 & 1,0170 \\
\hline 143 & 0,10 & 5,3 & 0,36 & 0,440 & 0,02 & 1,0355 & 1,0110 & 1,0240 & 0,838 & 1,0101 \\
\hline 144 & 0,15 & 5,3 & 0,36 & 0,580 & 0,02 & 1,0354 & & & 0,708 & 1,0050 \\
\hline 145 & 0,20 & 5,3 & 0,36 & 0,685 & 0,02 & 1,0355 & 1,0143 & 1,0206 & 0,628 & 1,0009 \\
\hline 146 & 0,30 & 5,3 & 0,36 & 0,860 & 0,02 & 1,0355 & & & 0,522 & 0,9934 \\
\hline 170 & 0,40 & 5,3 & 0,36 & 1,000 & 0,02 & 1,0354 & & & 0,456 & 0,9862 \\
\hline 114 & 0,50 & 5,3 & 0,36 & 1,100 & 0,02 & 1,0354 & 1,0200 & 1,0151 & 0,417 & 0,9788 \\
\hline 176 & 0,60 & 5,3 & 0,36 & 1,125 & 0,02 & 1,0356 & & & 0,412 & 0,9713 \\
\hline 177 & 0,70 & 5,3 & 0,36 & 1,040 & 0,02 & 1,0356 & & & 0,451 & 0,9647 \\
\hline 113 & 0,80 & 5,3 & 0,36 & 0,750 & 0,02 & 1,0354 & 1,0190 & 1,0161 & 0,610 & 0,9619 \\
\hline 122 & 0,85 & 5,3 & 0,36 & 0,500 & 0,02 & 1,0356 & & & 0,812 & 0,9630 \\
\hline 115 & 0,90 & 5,3 & 0,36 & 0,220 & 0,02 & 1,0354 & 1,0153 & 1,0202 & 1,168 & 0,9661 \\
\hline 199 & 0,10 & 5,3 & 0,36 & 0,405 & 0,40 & 1,0355 & 1,0114 & 1,0238 & 0,876 & 1,0043 \\
\hline 200 & 0,50 & 5,3 & 0,36 & 1,040 & 0,40 & 1,0355 & 1,0203 & 1,0151 & 0,442 & 0,9733 \\
\hline 201 & 0,60 & 5,3 & 0,36 & 1,080 & 0,40 & 1,0354 & 1,0212 & 1,0139 & 0,429 & 0,9659 \\
\hline 202 & 0,90 & 5,3 & 0,36 & 0,170 & 0,40 & 1,0355 & 1,0152 & 1,0200 & 1,253 & 0,9611 \\
\hline 175 & 0,10 & 5,05 & 0,36 & 0,515 & 0,02 & 1,0356 & 1,0113 & 1,0240 & 0,883 & 1,0095 \\
\hline 162 & 0,50 & 5,05 & 0,36 & 1,200 & 0,02 & 1,0354 & 1,0201 & 1,0150 & 0,439 & 0,9781 \\
\hline 165 & 0,90 & 5,05 & 0,36 & 0,290 & 0,02 & 1,0355 & 1,0158 & 1,0194 & 1,225 & 0,9654 \\
\hline 184 & 0,10 & 5,05 & 0,525 & 0,270 & 0,02 & 1,0354 & 1,0103 & 1,0251 & 0,868 & \\
\hline 193 & 0,50 & 5,05 & 0,525 & 0,875 & 0,02 & 1,0355 & 1,0199 & 1,0153 & 0,432 & 0,9803 \\
\hline 197 & 0,90 & 5,05 & 0,525 & 0,060 & 0,02 & 1,0355 & 1,0141 & 1,0211 & 1,226 & 0,9680 \\
\hline \multicolumn{6}{|c|}{ Experimentelle Werte: } & 1,0355 & $\begin{array}{r}1,0290 \\
-0,0066)\end{array}$ & 1,0063 & & \\
\hline
\end{tabular}

Tab. 3. Berechnete Isotopeneffekte. $f_{\mathrm{HCJ}}=0,205 \mathrm{mdyn} \AA$. Alle hier nicht besonders erwähnten Kraftkonstanten besitzen die in Tab. 1 genannten Werte.

Aus den angepaßten Kraftkonstanten-Sätzen, die den experimentellen ${ }^{13} \mathrm{C}$-Isotopeneffekt richtig wiedergeben, erhält man ungewöhnlich tiefe Werte für den D-Isotopeneffekt. Diese entsprechen den auf Grund des experimentellen C-Effektes ziemlich hoch zu wählenden Kraftkonstanten $f_{\mathrm{HCO}}$. Unseres Wissens liegen alle bisher gemessenen sekundären $\alpha$-Deuterium-Effekte bei $\mathrm{S}_{\mathrm{N}}$-Reaktionen zwischen 0,8 und 1,1 (siehe die Zusammenstellung in der vorhergehenden Arbeit ${ }^{2}$ sowie weitere Referenzen ${ }^{14,15}$ ). Es ist wenig wahrscheinlich, daß bei der Reaktion

14 K. T. Leffek, Can. J. Chem. 42, 851 [1964]

15 B. Östman, J. Am. Chem. Soc. 87, 3163 [1965]. von $\mathrm{CH}_{3} \mathrm{~J}$ mit $\mathrm{OH}^{-}$ganz andere Verhältnisse vorliegen.

Eine andere Möglichkeit, einen kleinen ${ }^{13} \mathrm{C}$-Isotopeneffekt zu berechnen, ohne die Deformationskraftkonstanten $f_{\mathrm{HCO}}$ und $f_{\mathrm{HCJ}}$ zu erhöhen, besteht in der Annahme eines unsymmetrischen Kraftfeldes für den Übergangszustand, in welchem $f_{\mathrm{CO}}$ und $f_{\mathrm{CJ}}$ stark voneinander abweichen ${ }^{3,16}$. In gleicher Weise wie bei der Behandlung der WasserstoffübertragungsDreizentrensysteme A ...H ... B ${ }^{17}$ wird hier ein Parameter $x$ definiert, der das Ausmaß von Bin-

16 F. H. Westheimer, Chem. Revs. 61, 265 [1961]. - J. Bigeleisen, Pure Appl. Chem. 8, 217 [1964].

17 A. V. Willi u. M. Wolfsberg, Chem. Ind. London 1964, 2097. 
dungsbruch und Bindungsbildung im Übergangszustand beschreiben soll. In einem Extremfall $(x=0)$ ist die CJ-Bindung noch gar nicht gebrochen, während die CO-Bindung gerade gebildet wird: $f_{\mathrm{CJ}}$ hat den gleichen Wert wie in $\mathrm{CH}_{3} \mathrm{~J}$, und für $f_{\mathrm{CO}}$ ist ein negativer Wert einzusetzen. (Das letztere bedeutet, daß der Übergangszustand ein Energiemaximum in bezug auf $\Delta r_{C O}$ darstellt.) Der andere Extremfall $(x=1)$ entspricht dem CJ-Bindungsbruch als Reaktionskoordinate, wobei $f_{\mathrm{CJ}}$ negativ zu wählen ist, während $f_{\mathrm{CO}}$ bereits den Wert der stabilen Bindung besitzt. Alle dazwischenliegenden Fälle sind als Linearkombinationen der beiden Extreme zu betrachten:

$$
\begin{gathered}
f_{\mathrm{CO} O}=4,5 x+a(1-x) \quad \operatorname{mdyn} / \AA, \\
f_{\mathrm{CJ}}=2,25(1-x)+b x \quad \operatorname{mdyn} / \AA, \\
(a<0 \text { und } b<0) .
\end{gathered}
$$

Zweckmäßigerweise hält man $d$ [Gl. (1)] konstant, wenn man den Isotopeneffekt als Funktion von $x$ betrachten will. Durch diese Bedingung ist die Wechselwirkungskraftkonstante $f_{12}$ nun ebenfalls festgelegt und kann mit Hilfe von Gl. (1) aus $d$, $f_{\mathrm{CO}}$ und $f_{\mathrm{CJ}}$ berechnet werden. Aus dem zusätzlichen Postulat, das $f_{12}$ sowohl für $x=0$ als auch für $x=1$ verschwinden soll (aber nicht im Bereich dazwischen), ergibt sich ein Zusammenhang zwischen $d, a$ und $b$ :

$$
d=2,25 a=4,50 b \mathrm{mdyn}^{2} / \AA^{2} .
$$

In den hier ausgeführten Proberechnungen ist $d=-5,40 \mathrm{mdyn}^{2} / \AA^{2}$ gesetzt. (Später - nach Beschaffung von weiterem experimentellem Material zum Vergleich - wären noch andere Möglichkeiten zu betrachten.)

In der ersten Gruppe der Daten in Tab. 3 (Nr. 137-103) sind die Isotopeneffekte für verschiedene $x$-Werte berechnet, wobei $f_{\mathrm{HCO}}$ und $f_{\mathrm{HCJ}}$ sowie alle anderen Kraftkonstanten (ausgenommen $f_{\mathrm{CO}}, f_{\mathrm{CJ}}$ und $f_{12}$ ) konstant gehalten werden. Wie zu erwarten, steigt $k_{12} / k_{13}$ anfänglich, durchläuft dann ein breites Maximum, um bei hohen $x$ wieder abzusinken. Interessanterweise muß der Übergangszustand verhältnismäßig stark „unsymmetrisch“ sein, wenn wesentlich tiefere ${ }^{13} \mathrm{C}$-Isotopeneffekte als der Maximalwert erhalten werden sollen. Der D-Isotopeneffekt ändert sich nur wenig als Funktion von $x$, denn die Kraftkonstanten-Änderungen finden ja hierbei nicht an der Position der Isotopensubstitution statt (siehe hierzu Ref. ${ }^{5}$ ). Der ${ }^{18} \mathrm{O}$-Isotopeneffekt ist deutlich von $x$ abhängig, da $f_{C O}$ mit wachsendem $x$ steigt. Demzufolge sinkt $k_{016} / k_{018}$ von einem Wert wenig größer als 1 (aber noch unterhalb $\nu_{\mathrm{L}, 16}^{\neq} / \nu_{\mathrm{L}, 18}^{\neq}$ auf ca. 0,96 . Bei $x=0,90$ befindet sich ein flaches Minimum.

In der zweiten Gruppe der Daten in Tab. 3 (Nr. 142-115) ist für jeden $x$-Wert $f_{\mathrm{HCO}}$ so angepaßt, daß der experimentelle ${ }^{13} \mathrm{C}$-Isotopeneffekt resultiert. Die aus diesen Kraftkonstanten-Sätzen berechneten D-Isotopeneffekte liegen zwischen 0,8 und 1,1 für die $x$-Bereiche um $0,05-0,10$ und um $0,85-0,90$. Der ${ }^{18} \mathrm{O}$-Isotopeneffekt zeigt im wesentlichen die gleiche Abhängigkeit von $x$ wie in der ersten Gruppe der Daten, wenn auch Abweichungen von wenigen Promille vorkommen. Einige weitere, in diesen Tabellen nicht wiedergegebenen Proberechnungen bestätigen, daß der Einfluß von $f_{\mathrm{OH}}$ gering ist, solange diese Kraftkonstante im Bereich ihrer wahrscheinlichen Werte bleibt. Eine Erniedrigung von 7,45 auf 7,30 mdyn/ $\AA$ bewirkt eine Erhöhung von $k_{016} / k_{018}$ um $0,03 \%$.

In der dritten Gruppe der Daten in Tab. 3 $(\mathrm{Nr} .198-202)$ ist $f_{\mathrm{COH}}=0,40 \mathrm{mdyn} / \AA$ gesetzt und $f_{\text {HCO }}$ wiederum auf den experimentellen C-Effekt angepaßt. Die D-Effekte sind $3-5 \%$ höher und die O-Effekte sind $0,6 \%$ tiefer als in den entsprechenden Rechnungen der zweiten Gruppe.

Eine mögliche gehinderte Rotation des freien Hydroxid-Ions in Lösung sollte sich hier auf $k_{016} / k_{018}$ kaum auswirken, da der Schwerpunkt nahe beim O-Atom liegt, so daß die Trägheitsmomente von ${ }^{16} \mathrm{OH}^{-}$und ${ }^{18} \mathrm{OH}^{-}$nur wenig voneinander abweichen.

Resultate von Proberechnungen, die denjenigen der zweiten Gruppe entsprechen, sind ferner vorhanden für andere Werte von $f_{\mathrm{CH}}$ und $f_{\mathrm{HCH}}$, nämlich $5,05 \mathrm{mdyn} / \AA$ und $0,36 \mathrm{mdyn} \AA$ sowie $5,05 \mathrm{mdyn} / \AA$ und 0,525 mdyn $\AA$. Aus Gründen der Platzersparnis werden von diesen nur wenige Beispiele angeführt. Es sind hierbei andere Werte für $f_{\mathrm{HCO}}$ einzusetzen, doch die berechneten D- und O-Effekte bleiben nahezu die gleichen, da jeweils eine Änderung von $f_{\mathrm{CH}}$ oder $f_{\mathrm{HCH}}$ ( - soweit sie nicht zu stark ist - ) durch eine entgegengerichtete Änderung von $f_{\mathrm{HCO}}$ kompensiert wird.

Selbstverständlich haben die hier verwendeten $x$-Werte nur relative Bedeutung. Sie sind nur dann vollkommen definiert, wenn das Schwingungsproblem des Übergangszustandes immer in der gleichen Weise formuliert wird unter Beibehaltung der Geometrie und der Werte für $d, f_{\mathrm{CH}}, f_{\mathrm{HCH}}, g$ und die 
4. Wechselwirkungskonstanten innerhalb der $\mathrm{CH}_{3}$ Gruppierung. Vor allem der Einfluß von $d$ ist bedeutend: Bei schwach negativem $d$ steigt $k_{12} / k_{13}$ an beiden Seiten schwächer an, und das Maximum in der Mitte ist schmaler. Ein stärker negatives $d$ verbreitert den $x$-Bereich des Maximums von $k_{12} / k_{13}$, so daß der Anstieg an beiden Seiten größer wird ${ }^{17}$.

\section{Die Temperaturabhängigkeit des Kohlenstoff-13-Isotopeneffektes}

Wie in der vorhergehenden Arbeit wird auch hier auf die für 5 Temperaturen (im Bereich von $284,6^{\circ}-331,2^{\circ} \mathrm{K}$ ) berechneten ${ }^{13} \mathrm{C}$-Isotopeneffekte die Methode der kleinsten Quadrate angewandt zur Berechnung der Regressionsgraden von $\ln \left(k_{12} / k_{13}\right)$ in Abhängigkeit von $1 / T$. Auf diese Weise erhält man Arrhenius-Parameter des berechneten Isotopeneffektes gemäß Gl. (4), die auf einen beschränkten Temperaturbereich anwendbar sind und sich für den Vergleich mit den Arrhenius-Parametern des gemessenen Isotopeneffektes eignen (siehe die Tab. 2 und 3)

$k_{12} / k_{13}=A \cdot \exp \left(-\Delta \Delta{E^{\prime}}_{0} / R T=A \cdot(A Z P E){ }^{17 \mathrm{a}}\right.$

Die berechneten $A$-Werte sind alle etwas niedriger als der experimentelle $A$-Wert (Tab. 3 ), wobei der höchste gerade ungefähr eine Standard-Abweichung tiefer liegt. Die Mehrzahl der anderen befinden sich noch innerhalb der zweiten Standardabweichung. In Tab. 3 zeigen die berechneten $A$-Werte eine deutliche Abhängigkeit von $x$, - die Unterschiede sind aber nicht wesentlich größer als die Standardabweichung des bis jetzt bekannten experimentellen Wertes. Zudem können die berechneten $A$-Werte ( - auch diejenigen bei $x=0,5-0,6-$ ) durch Wahl eines anderen $d$ um 1-2 Promille verändert werden (vgl. z. B. Nr. 8 und 4 in Tab. 2). (Hierbei wird vorausgesetzt, daß $d$ im Bereich von 0 bis ca. -8 bleibt. Noch stärker negative $d$-Werte dürfen als unrealistisch betrachtet werden.)

Die Temperaturabhängigkeit der D- und ${ }^{18} \mathrm{O}$-Isotopeneffekte soll in dieser Arbeit noch nicht diskutiert werden. Sobald einmal experimentelle Daten zum D-Isotopeneffekt vorliegen, dann wird es möglich sein, durch Vergleich von berechneten und experimentellen ArRhenius-Parametern des D-Effektes Schlüsse in bezug auf die zu verwendenden Werte für die Kraftkonstanten $f_{\mathrm{CH}}$ und $f_{\mathrm{HCH}}$ zu ziehen.

17a $(A Z P E)$ bedeutet "apparent zero point energy factor" 5.
Eine Diskussion dieser Zusammenhänge ist bereits für den Übergangszustand $\mathrm{J} \ldots \mathrm{CH}_{3} \ldots \mathrm{J}$ durchgeführt worden ${ }^{1}$.

\section{Schlußfolgerungen}

Aus den in dieser Arbeit durchgeführten Proberechnungen im Vergleich $\mathrm{zu}$ den vorliegenden experimentellen Daten lassen sich wichtige Schlüsse von allgemeiner organisch-chemischer Bedeutung ziehen. So folgt aus dem verhältnismäßig kleinen experimentellen Wert des ${ }^{13} \mathrm{C}$-Isotopeneffektes, daß der Übergangszustand der Reaktion von $\mathrm{CH}_{3} \mathrm{~J}$ mit $\mathrm{OH}^{-}$„unsymmetrisch“ sein muß, d. h. es gilt entweder $f_{\mathrm{CO}}>f_{\mathrm{CJ}}$ oder $f_{\mathrm{CO}}<f_{\mathrm{CJ}}$ (Abweichungen von $3,5-5$ mdyn $/ \AA)$, - sofern der bisher noch nicht gemessene D-Isotopeneffekt wirklich in den Bereich von $k_{\mathrm{H}} / k_{\mathrm{D}}=0,8-1,1$ fällt.

Sobald einmal experimentelle Daten für alle drei Isotopeneffekte in diesem System vorliegen, kann man folgendermaßen vorgehen: Zunächst wird an Hand von Proberechnungen die Summe der Deformationskraftkonstanten $f_{\mathrm{HCO}}+f_{\mathrm{HCJ}}$ (bzw. $f_{\mathrm{HCO}}$ bei festgehaltenem $f_{\mathrm{HCJ}}$ ) des Übergangszustandes ermittelt, die den gemessenen D-Isotopeneffekt $k_{\mathrm{H}} / k_{\mathrm{D}}$ richtig wiedergibt. Im nächsten Schritt werden mit Hilfe des gefundenen Resultates für $f_{\mathrm{HCO}}+f_{\mathrm{HCJ}}$ die beiden $x$-Werte (zu beiden Seiten des Maximums) gesucht, die den experimentellen C-Isotopeneffekt $k_{12} / k_{13}$ reproduzieren. Der gemessene O-Isotopeneffekt $k_{016} / \mathrm{k}_{018}$ im Vergleich mit den berechneten Werten sollte schließlich eine Entscheidung darüber ermöglichen, auf welcher Seite sich die „Unsymmetrie" des Übergangszustandes befindet, d. h. welcher der beiden $x$-Werte (größer als 0,6 oder kleiner als 0,6) wirklich in Frage kommt.

So kann die Information, welche experimentelle Daten von kinetischen Isotopeneffekten zu liefern vermögen, viel besser als bisher ausgewertet werden, wenn man quantitative Proberechnungen mit Kraftkonstanten als Ausgangsdaten zum Vergleich durchführt.

Der Autor dankt Herrn Dr. Max Wolfsberg, Department of Chemistry, Brookhaven National Laboratory, für die freundliche Überlassung von Maschinenprogrammen sowie für viele wertvolle und interessante Diskussionen. Er dankt ferner dem Computer Center der "Columbia University in the City of New York" für die Gewährung von Maschinenzeit. 